\title{
A Study of the Conformational Isomerism of 1-Iodobutane by High Resolution Rotational Spectroscopy
}

\author{
Eric A. Arsenault ${ }^{\mathrm{a}}$, Daniel A. Obenchain ${ }^{\mathrm{a}, \mathrm{b}}$, Thomas A. Blake ${ }^{\mathrm{c}}$, S. A. Cooke $^{\mathrm{d}}$, \\ Stewart E. Novick ${ }^{\mathrm{a}}$ \\ ${ }^{a}$ Department of Chemistry, Wesleyan University, Hall-Atwater Laboratories, 52 Lawn Ave, \\ Middletown, CT 06459 \\ ${ }^{b}$ Present Address: Institut für Physikalische Chemie und Elektrochemie, Lehrgebiet A, \\ Gottfried-Wilhelm-Leibniz-Universität, Callinstrasse 3A, D-30167 Hannover, Germany \\ ${ }^{c}$ Pacific Northwest National Laboratory, 902 Battelle Blvd, Richland, WA 99354 \\ ${ }^{d}$ School of Natural and Social Sciences, Purchase College SUNY, 735 Anderson Hill Rd, \\ Purchase, NY 10577
}

\begin{abstract}
The first microwave study of 1-iodobutane, performed by Steinmetz et al. in 1977 , led to the determination of the $B+C$ parameter for the anti-antiand gauche-anti-conformers. Nearly 40 years later, this reinvestigation of 1iodobutane, by high-resolution microwave spectroscopy, led to the determination of rotational constants, centrifugal distortion constants, nuclear quadrupole coupling constants (NQCCs), and nuclear-spin rotation constants belonging to both of the two previously mentioned conformers, in addition to the gauchegauche-conformer, which was observed in this frequency regime for the first time. Comparisons between the three conformers of 1-iodobutane and other iodo- and bromoalkanes are made, specifically through an analysis of the nuclear quadrupole coupling constants belonging to the iodine and bromine atoms in the respective chemical environments.
\end{abstract}

Keywords: rotational spectroscopy, nuclear quadrupole coupling tensor, conformations, iodine, haloalkane, FTMW

\footnotetext{
${ }^{*}$ Corresponding author

Email address: snovick@wesleyan.edu (Stewart E. Novick)
}

Preprint submitted to Journal of Molecular Spectroscopy

March 16, 2017

(C) 2017. This manuscript version is made available under the Elsevier user license http://www.elsevier.com/open-access/userlicense/1.0/ 


\section{Introduction}

It has been commonly accepted that the electric field gradient of a nucleus remains unchanged in most simple molecules even as they take on different conformations or as they form van der Waals complexes. A field gradient caused

${ }_{5}$ by a charge is proportional to $1 / r^{3}$, where $r$ is the distance from the charge to the nucleus. It is presented by Townes and Dailey[1] and outlined in Gordy and Cook[2], how the field gradient found at a nucleus can be assumed to have been produced by the bonds made by that atom. This analysis was originally for bonding $p$ orbitals, but was later extended to include hybrid orbital contributions by Novick in 2011[3]. This unchanging nature of a field gradient was recently used to correctly determine the structure of $\mathrm{HOD}-\mathrm{N}_{2} \mathrm{O}[4]$.

The assumption that the field gradient will remain unchanged upon the formation of a complex, conformational change, or isotopic substitution has, of course, exceptions. Choosing $\mathrm{N}_{2} \mathrm{O}$ and the complexes it forms as an example[515 16], it was shown repeatedly that there are sublte changes in the electronic environment of near atoms as complexes are formed. For $\mathrm{HCCH} \cdots \mathrm{N}_{2} \mathrm{O}[5,7]$, as studied by Leung and coworkers, it was shown that in forming the complex there is a significant change in the electric field gradient of the central nitrogen, while the field gradient at terminal nitrogen remained unchanged. Through molecular multipole analysis, the authors showed that this change is caused by redistribution of electrons about the central nitrogen.

To observe changes in electronic structure from perturbations smaller in magnitude than those observed upon forming a van der Waals complex, such as conformational changes, a more sensitive nucleus is required to act as a probe

25 for this change. Iodine, with its large nuclear electric quadrupole moment, -69.6(12) $\mathrm{fm}^{2}$ [17] for ${ }^{127} \mathrm{I}$, compared to the ${ }^{14} \mathrm{~N}$ value of $2.001(10) \mathrm{fm}^{2}[18]$, makes the observed nuclear quadrupole coupling constants significantly more sensitive to small changes in electron distribution near the iodine nucleus. For example, in a recent study of iodobenzene and the Ne-iodobenzene complex[19], there is a $<0.3 \%$ change in the iodine NQCC upon forming the complex with 
neon. We have recently reported a small, but significant, change in electronic structure from carbon-13 isotopic substitution in 2-iodobutane[20].

Continuing a series of studies on the subtle changes in electric structure determined by changes in nuclear quadrupole coupling constants, we report

35 here on the conformational effects on a terminal iodine group in a hydrocarbon chain.

\section{Experimental}

The high-resolution rotational spectrum of 1-iodobutane was measured from 7-13 GHz with a chirped-pulse Fourier transform microwave (FTMW) spectrom-

40 eter. Detailed specifications of this spectrometer, which is based on the design of Pate and coworkers[21], have been presented previously[22]. In short, a chosen microwave center frequency, $\nu$, and a $6 \mu$ s linear frequency sweep are mixed from $\mathrm{DC}$ to $1 \mathrm{GHz}$. The resulting radiation, $\nu \pm 1 \mathrm{GHz}$, is broadcast directly into a vacuum chamber through a microwave horn antenna. This transmitted

45 radiation then induces a polarization in the coincident molecular beam. A second microwave horn antenna collects the free induction decay (FID) after a $1 \mu \mathrm{s}$ delay. With the aid of a Tektronix TDS6124C Digital Oscilloscope, the FID is fast Fourier transformed and directly digitized. A total of 800,000 points, over a time of $20 \mu \mathrm{s}$, are collected from the FID. On average, the molecular rotational transitions have a line width of $80 \mathrm{kHz}$ with an uncertainty of $|8| \mathrm{kHz}$ in the center frequency.

The sample was acquired from Sigma-Aldrich ${ }^{\circledR}\left(\geq 99 \% \mathrm{CIH}_{2} \mathrm{CH}_{2} \mathrm{CH}_{2} \mathrm{CH}_{3}\right)$. Further purification was not necessary. The sample (bp $130-131{ }^{\circ} \mathrm{C}$ ) was contained in a glass U-form tube at room temperature and one atmosphere of dry ${ }_{55} \operatorname{argon}\left(99.999 \%\right.$, Airgas $\left.{ }^{\circledR}\right)$ was bubbled directly through the liquid. The final mixture of carrier gas and sample was pulsed through a solenoid valve into the chamber, held at an ambient pressure of $10^{-6}$ Torr, allowing the molecules to undergo supersonic expansion, where the molecules become rotationally cold (1-2 K). 
Using a $321 \mathrm{G}^{*}$ basis set at the APFD level of theory, a coordinate scan of the $\mathrm{C}-\mathrm{C}-\mathrm{C}-\mathrm{C}$ and $\mathrm{C}-\mathrm{C}-\mathrm{C}-\mathrm{I}$ dihedral angles was performed, in order to identify the most probable ground state molecular geometries. Three of the lowest energy structures from the scan were optimized at the MP2 level of theory. A 6311G* basis set was imported from the EMSL Basis Set Library[23, 24] specifically chosen to handle the iodine atom, while a $6311 \mathrm{G}++(2 \mathrm{~d}, 2 \mathrm{p})$ basis set was used for the remaining carbon and hydrogen atoms. All calculations were performed with the GAUSSIAN09 Revision D suite[25]. Results from the ab initio optimizations are presented in Table 1. Illustrations of the corresponding structures can be found in Figure 1. More on predicting the NQCCs will be discussed in the subsequent sections.

Table 1: Rotational constants and NQCCs for three conformers of 1-iodobutane as determined from $a b$ initio optimization at the MP2 level of theory.

\begin{tabular}{|c|c|c|c|}
\hline Parameters & $\mathrm{gg}$ & ga & aa \\
\hline$A(\mathrm{MHz})$ & 6033 & 7388 & 15123 \\
\hline$B(\mathrm{MHz})$ & 1077 & 937 & 706 \\
\hline$C(\mathrm{MHz})$ & 1013 & 867 & 686 \\
\hline${ }^{127} \mathrm{I}_{\chi_{a a}}(\mathrm{MHz})$ & -397 & -274 & -682 \\
\hline${ }^{127} \mathrm{I}_{\chi_{b b}}(\mathrm{MHz})$ & 74 & -167 & 223 \\
\hline${ }^{127} \mathrm{I}_{\chi_{c c}}(\mathrm{MHz})$ & 322 & 441 & 459 \\
\hline${ }^{127} \mathrm{I}_{\chi_{a b}}(\mathrm{MHz})$ & -570 & -670 & -511 \\
\hline${ }^{127} \mathrm{I} \chi_{a c}(\mathrm{MHz})$ & -330 & -109 & $0^{b}$ \\
\hline${ }^{127} \mathrm{I}_{\chi_{b c}}(\mathrm{MHz})$ & -226 & -104 & 0 \\
\hline$\Delta \mathrm{E}^{a}\left(\mathrm{~cm}^{-1}\right)$ & 385 & 160 & 0 \\
\hline
\end{tabular}

${ }^{a} \overline{\overline{\text { Relative energies from MP2 optimization }}}$.

${ }^{b} \chi_{a c}$ and $\chi_{b c}$ are zero by symmetry. 


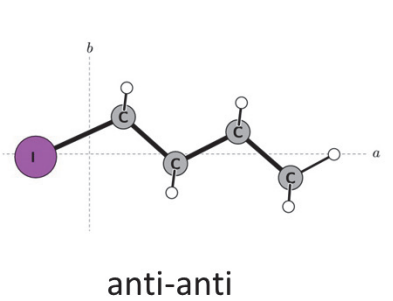

(aa)

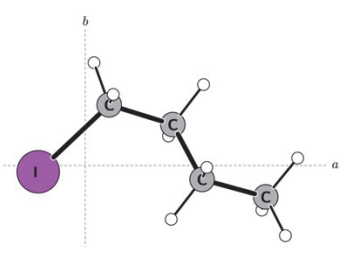

gauche-anti

(ga)

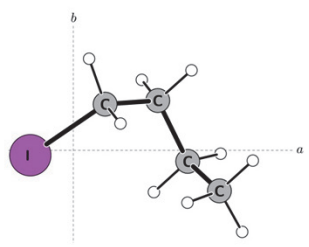

gauche-gauche

(gg)

Figure 1: Calculated structures of the anti-anti (aa)-, gauche-anti (ga)-, and gauche-gauche (gg)-conformers of 1-iodobutane from an ab initio optimization. The $\mathrm{C}-\mathrm{C}-\mathrm{C}-\mathrm{C}$ and $\mathrm{C}-\mathrm{C}-\mathrm{C}-\mathrm{I}$ dihedral angles for the aa-, ga-, and gg-species were calculated to be $180^{\circ}, 180^{\circ}$; $179^{\circ}, 66^{\circ}$; and $-65^{\circ},-63^{\circ}$.

Spectral Assignments

All three of the lowest energy conformers obtained from the $a b$ initio investigation were successfully assigned from the rotational spectrum collected in the frequency range of 7-13 GHz. A $70 \mathrm{MHz}$ portion of this spectrum is shown in Figure 2. The three broadband assignments were accomplished with the help of both the AABS package[26] and Pickett's programs, SPFIT/SPCAT[27, 28]. The final rotational constants, centrifugal distortion constants, NQCCs, and nuclear spin-rotation constants for each conformer can be found in Table 2 . Although there is a large discrepancy between the ab initio and experimental NQC tensors belonging to the iodine atom in each of the three species, there is at most only a $7 \%$ difference between the rotational constants obtained from the $a b$ initio study and those determined experimentally. This seems to suggest that the actual geometries of the three conformers present in the molecular beam are quite similar to those that were calculated, whereas the only agreement in the NQCCs was in the trend of their respective magnitudes.

The poor ab initio NQCC values made the first assignment, of the gaconformer, quite challenging. In order to make the assignment process more efficient and less tedious, alternate methods of prediction were employed. Tak-

ing advantage of the fact that changes in the geometry of the butane chain have 


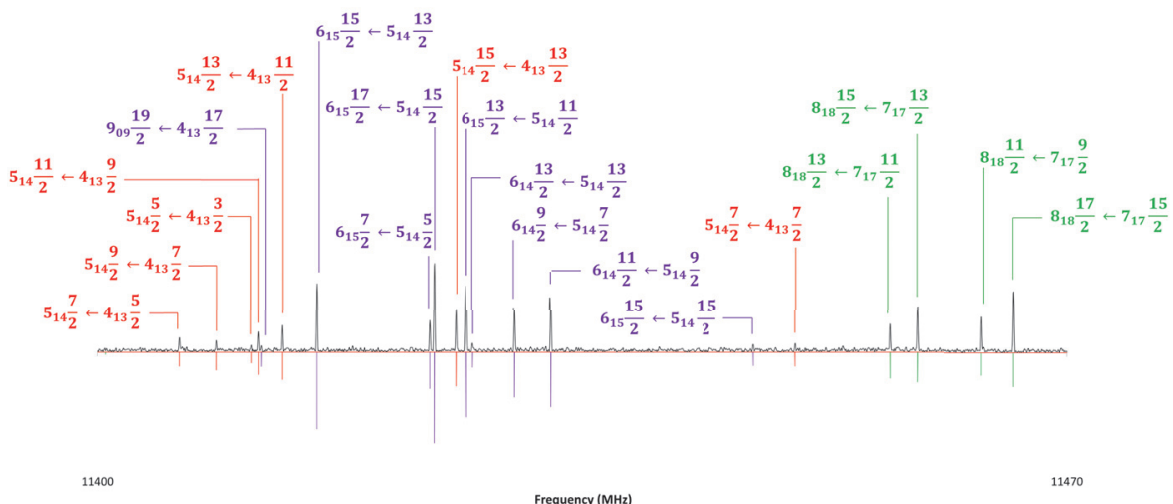

Figure 2: A small portion of the experimental spectrum of 1-iodobutane is shown in black, with the final simulated spectra for the aa-, ga-, and gg-conformers shown below in green, purple, and red, respectively. The quantum numbers associated with each rotational transition are also presented above the experimental spectrum, in the form $J_{K_{a}^{\prime} K_{c}^{\prime}}^{\prime} F^{\prime} \leftarrow J_{K_{a}^{\prime \prime} K_{c}^{\prime \prime}}^{\prime \prime} F^{\prime \prime}$.

\section{Prediction using}

hybrid tensor:

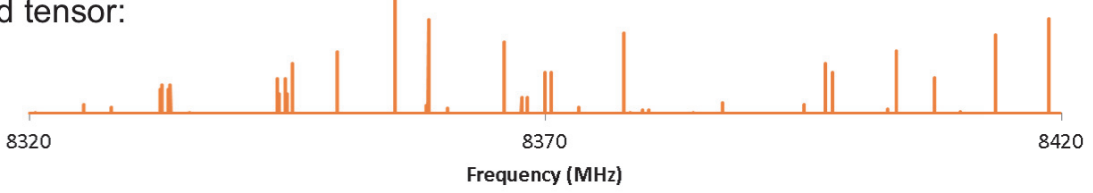

Prediction

from fit:
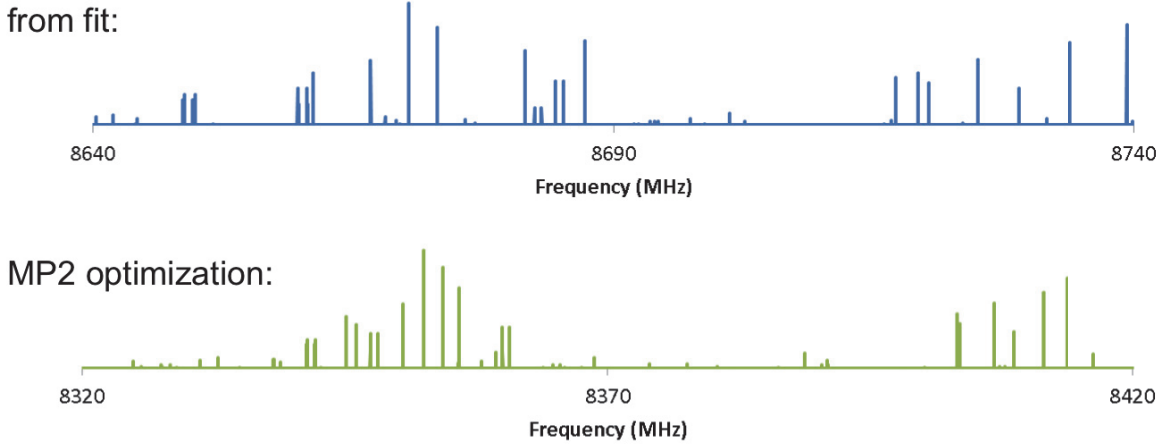

Figure 3: A $100 \mathrm{MHz}$ portion of three different predictions of the rotational spectrum of aa1-iodobutane. The top orange spectrum is based on a hybrid tensor (discussed in Spectral Assignments) and $a b$ initio rotational constants, the middle blue spectrum is the prediction using the experimental results belonging to the aa-conformer, and the bottom orange spectrum is based completely on ab initio results. 


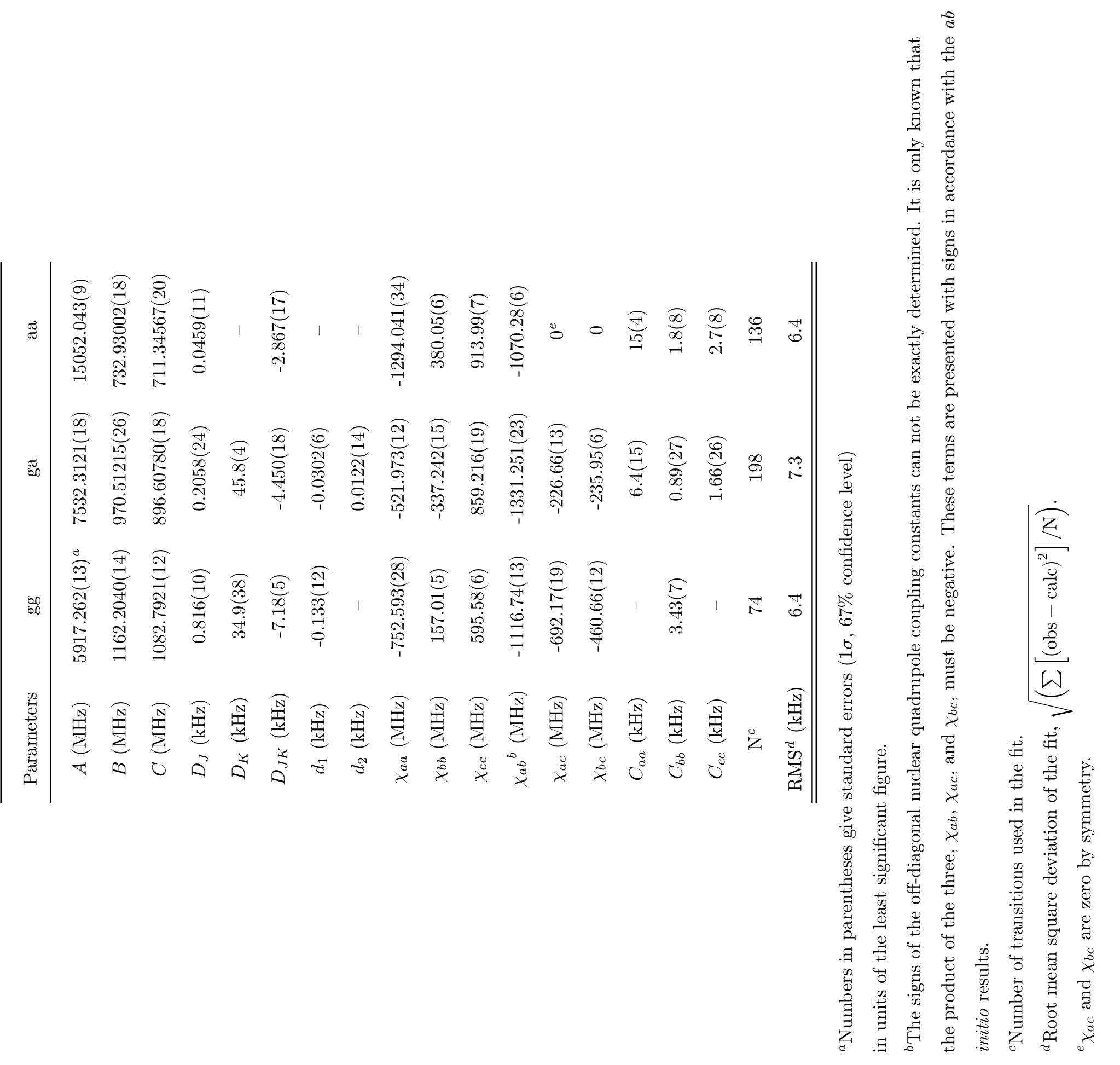


a very small effect on the electric field gradient at the iodine nucleus (more on this to come later), the experimental NQC tensor of iodine in the ga-conformer was used to make predictions of the NQC tensors of iodine in both of the two remaining unassigned conformers. This predictive process was simply an exercise in tensor rotation. Using QDIAG[29], the ab initio tensors of the two unassigned conformers were diagonalized. In doing this, the rotation matrices, specific to the $a b$ initio geometries of these species, were obtained. Then, these respective rotation matrices were used to transform the experimental NQC tensor of the ga-conformer into the inertial axes systems of the gg- and aa-conformers. The resulting hybrid tensors were based on ab initio geometries, specific to the unassigned conformers, and experimental NQCCs, belonging to the already assigned ga-conformer. To best illustrate the power of this method, the NQC tensor predictions of the aa-conformer will used as an example. Equation (1) contains the $a b$ initio tensor belonging to the aa-conformer, Equation (2) contains the hybrid tensor, and Equation (3) contains the experimental tensor based only on rotational transitions belonging to the aa-conformer. It is perhaps immediately obvious that the hybrid tensor, again based on the ab initio geometry of the aa-conformer and the rotated experimental NQC tensor of the ga-conformer, offers a much better prediction than the purely $a b$ initio tensor. To further highlight this point, Figure 3 presents small portions of the rotational spectra corresponding to these respective tensors. It is worth noting here that the similarities between the NQC tensors of iodine in various iodoalkanes, such as iodoethane, t-1-iodopropane, and aa-1-iodobutane, which will be discussed later, suggest that this method can be applied to quite a large range of problems. This method, based on straightforward linear algebra, can lead to very accurate predictions without the need for expensive computations.

It should be noted there are other methods used to make accurate predictions of NQQCs, especially for species with large quadrupoles, as shown by Professor W. C. Bailey[30]. Post-analysis, we received calculations from Professor Bai120 ley, which can be found in Table 3 alongside the predictions from our hybrid approach[31, 32]. These calculations involve the calibration of a specific com- 
bination of level of theory and basis set, by linear regression, of the calculated electric field gradients versus the experimental NQCCs of a selected group of molecules. Once calibrated, the specific combination of level of theory and basis set can be applied to other systems. Upon comparison of Table 2 and Table 3, it can be seen that this method yields excellent results. The differences between the hybrid method explained previously and these calculations are small. Although it is clear that these calculations are quite accurate, the hybrid method can rapidly provide good predictions based on very computationally inexpensive optimizations.

$$
\begin{gathered}
\chi_{M P 2}=\left(\begin{array}{ccc}
-682 & -511 & 0 \\
-511 & 223 & 0 \\
0 & 0 & 459
\end{array}\right) \\
\chi_{\text {hybrid }}=\left(\begin{array}{ccc}
-1346 & -1019 & 0 \\
-1019 & 462 & 0 \\
0 & 0 & 884
\end{array}\right) \\
\chi_{\text {exp }}=\left(\begin{array}{ccc}
-1294.041(34) & -1070.28(6) & 0 \\
-1070.28(6) & 380.05(6) & 0 \\
0 & 0 & 913.99(7)
\end{array}\right)
\end{gathered}
$$

\section{Theory}

The hyperfine structure in the rotational spectrum of 1-iodobutane is a consequence of the nuclear spin of iodine $\left(I=\frac{5}{2}\right)$, which allows for the observation of both nuclear quadrupole coupling and nuclear spin-rotation coupling. The respective Hamiltonians, which account for these interactions, were combined with both the rigid rotor and centrifugal distortion Hamiltonians. The final form of the Hamiltonian then becomes[20, 33-35]:

$$
\hat{\mathcal{H}}=\hat{\mathcal{H}}_{R}+\hat{\mathcal{H}}_{C D}+\hat{\mathcal{H}}_{Q}+\hat{\mathcal{H}}_{S R}
$$


Table 3: Comparison of the two methods of prediction for the NQC tensors in 1-iodobutane.

\begin{tabular}{|c|c|c|c|c|c|c|}
\hline \multirow[b]{2}{*}{ Parameters } & \multicolumn{3}{|c|}{$\mathrm{MP} 2 / 6311+\mathrm{G}(\mathrm{d}, \mathrm{p})^{a}$} & \multicolumn{3}{|c|}{ Hybrid Method } \\
\hline & $\mathrm{gg}$ & ga & aa & $\mathrm{gg}$ & $\mathrm{ga}^{b}$ & aa \\
\hline${ }^{127} \mathrm{I}_{\mathrm{a} a}(\mathrm{MHz})$ & -757 & -546 & -1317 & -706 & - & -1346 \\
\hline${ }^{127} \mathrm{I}_{\chi_{b b}}(\mathrm{MHz})$ & 164 & -314 & 400 & 104 & - & 462 \\
\hline${ }^{127} \mathrm{I}_{\chi_{c c}}(\mathrm{MHz})$ & 593 & 860 & 917 & 602 & - & 884 \\
\hline${ }^{127} \mathrm{I} \chi_{a b}(\mathrm{MHz})$ & -1119 & -1336 & -1062 & -1025 & - & -1019 \\
\hline${ }^{127} \mathrm{I}_{\chi_{a c}}(\mathrm{MHz})$ & -702 & -234 & $0^{c}$ & -632 & - & $0^{c}$ \\
\hline${ }^{127} \mathrm{I}_{\chi_{b c}}(\mathrm{MHz})$ & -464 & -238 & 0 & -367 & - & 0 \\
\hline
\end{tabular}

${ }^{a}$ These calculations were performed by Professor W. C. Bailey with calibrated MP2/6311+G(d,p) combination[31, 32].

${ }^{b}$ These values were not predicted via the hybrid method, as the experimental NQC tensor of the ga-conformer was used to make the predictions for the other two conformers. Rather, the NQC tensor presented in Table 1 was used.

${ }^{c}$ Zero by symmetry. 
Quantum labels for the rotational transitions belonging to 1-iodobutane are as follows: $J_{K_{a}^{\prime} K_{c}^{\prime}}^{\prime} F^{\prime} \leftarrow J_{K_{a}^{\prime \prime} K_{c}^{\prime \prime}}^{\prime \prime} F^{\prime \prime}$, where $\boldsymbol{F}=\boldsymbol{I}+\boldsymbol{J}$. The quantum number $\boldsymbol{F}$ is the total angular momentum quantum number that accounts for the coupling between the nuclear spin of iodine and the rotational angular momentum of the molecule.

\section{Discussion}

Table 4: Comparison of this work with the previous study of 1-iodobutane.

\begin{tabular}{cccc}
\hline & Parameters & This work. & Steinmetz et al.[36] \\
\hline ga & $B+C(\mathrm{MHz})$ & $1867.11995(32)$ & $1868.4(30)^{a}$ \\
aa & $B+C(\mathrm{MHz})$ & $1444.27569(27)$ & $1445.3(4)$ \\
gg & $B+C(\mathrm{MHz})$ & $2244.9961(18)$ & Not observed. \\
\hline
\end{tabular}

${ }^{a}$ Numbers in parentheses give standard errors (1 $\sigma, 67 \%$ confidence level) in units of the least significant figure.

For the first time since 1977, 1-iodobutane was reinvestigated via microwave 145 spectroscopy. Table 4 presents a comparison between this study and the work of Steinmetz et al.[36] In the previous low-resolution microwave study, the $B+C$ parameter was measured for the ga- and aa-conformers. Between studies, the measured values of $B+C$ are within $0.07 \%$ for both the ga-conformer and aaconformer. In this study, the gg-conformer was spectroscopically detected in this frequency range for the first time, although previous work[36] did indicate that this species should likely be present in low abundance, as the highest energy conformer of the three.

Due to the flexible nature of this molecule, the three observed conformers possess quite different rotational constants. This is most evident upon noting that the difference between the $A$ rotational constant of the heavy atom planar aa-conformer and the highly asymmetric ga-conformer is over $9100 \mathrm{MHz}$, as seen in Table 2. As expected, the large structural differences between these 
Table 5: NQC tensor of iodine in 1-iodobutane.

\begin{tabular}{cccc}
\hline Parameters & gg & ga & aa \\
\hline Energies $\left(\mathrm{cm}^{-1}\right)^{a}$ & 385 & 160 & 0 \\
$\chi_{z z}(\mathrm{MHz})$ & $-1758.57(14)^{b}$ & $-1804.133(35)$ & $-1815.72(6)$ \\
& $-3.15 \%^{c}$ & $-0.64 \%$ & - \\
$\chi_{y y}(\mathrm{MHz})$ & $1182.40(19)$ & $920.04(9)$ & $901.73(7)$ \\
& $+31.13 \%$ & $+2.03 \%$ & - \\
$\chi_{x x}(\mathrm{MHz})$ & $576.17(16)$ & $884.09(11)$ & $913.99(7)$ \\
$\eta_{\chi}{ }^{d}$ & $-36.96 \%$ & $-3.27 \%$ & - \\
\hline \hline
\end{tabular}

${ }^{a}$ From Table 1.

${ }^{b}$ Numbers in parentheses give standard errors $(1 \sigma, 67 \%$ confidence level) in units of the least significant figure.

${ }^{c}$ Percentage change relative to the corresponding component of the lowest energy aa-conformer.

${ }^{d} \eta_{\chi}$ is a measure of the asymmetry of the nuclear quadrupole coupling tensor, where $\eta_{\chi}=\frac{\chi_{x x}-\chi_{y y}}{\chi_{z z}}$. 
Table 6: NQC tensor of bromine in 1-bromobutane[37].

\begin{tabular}{cccc}
\hline Parameters & gg & ga & aa \\
\hline${ }^{79} \mathrm{Br} \chi_{z z}(\mathrm{MHz})$ & $536.2(5)^{a}$ & $539.7(6)$ & $543.45(34)$ \\
${ }^{79} \mathrm{Br} \chi_{y y}(\mathrm{MHz})$ & $-264.9(6)$ & $-264.9(20)$ & $-269.5(6)$ \\
${ }^{79} \mathrm{Br} \chi_{x x}(\mathrm{MHz})$ & $-271.29(36)$ & $-274.8(15)$ & $-274.0(9)$ \\
$\eta_{\chi}{ }^{b}$ & $-0.0119(13)$ & $-0.018(5)$ & $-0.0083(21)$ \\
${ }^{81} \mathrm{Br} \chi_{z z}(\mathrm{MHz})$ & $448.3(47)$ & $451.1(9)$ & $453.95(35)$ \\
${ }^{81} \mathrm{Br} \chi_{y y}(\mathrm{MHz})$ & $-222.0(19)$ & $-220.2(23)$ & $-225.5(7)$ \\
${ }^{81} \mathrm{Br} \chi_{x x}(\mathrm{MHz})$ & $-226.3(39)$ & $-231.0(19)$ & $-228.4(9)$ \\
$\eta_{\chi}$ & $-0.01(1)$ & $-0.024(7)$ & $-0.0065(24)$ \\
\hline \hline
\end{tabular}

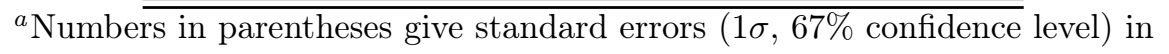
units of the least significant figure.

${ }^{b} \eta_{\chi}$ is a measure of the asymmetry of the nuclear quadrupole coupling tensor, where $\eta_{\chi}=\frac{\chi_{x x}-\chi_{y y}}{\chi_{z z}}$. 
Table 7: A comparison of this work with similar haloalkanes.

\begin{tabular}{|c|c|c|c|}
\hline & iodoethane[38] & t-1-iodopropane[39] & aa-1-iodobutane \\
\hline${ }^{127}{ }^{\mathrm{I}_{z z}}(\mathrm{MHz})$ & $-1815.22(85)^{a}$ & $-1814.55(55)$ & $-1815.72(6)$ \\
\hline${ }^{127} \mathrm{I}_{\chi_{y y}}(\mathrm{MHz})$ & $901.71(81)$ & $900.44(47)$ & $901.73(7)$ \\
\hline${ }^{127}{ }^{\mathrm{I}} \chi_{x x}(\mathrm{MHz})$ & $913.50(26)$ & $914.12(44)$ & $913.99(7)$ \\
\hline \multirow[t]{2}{*}{$\eta_{\chi}^{b}$} & $-0.0065(6)$ & $-0.0075(5)$ & $-0.00675(6)$ \\
\hline & bromoethane[38] & t-1-bromopropane[40] & aa-1-bromobutane[37] \\
\hline${ }^{79}{ }^{\mathrm{Br}} \chi_{z z}(\mathrm{MHz})$ & $544.03(168)$ & $541.6(7)$ & $543.45(34)$ \\
\hline${ }^{79} \mathrm{Br} \chi_{y y}(\mathrm{MHz})$ & $-270.32(166)$ & $-267.8(7)$ & $-269.5(6)$ \\
\hline${ }^{79}{ }^{7 r} \chi_{x x}(\mathrm{MHz})$ & $-273.71(17)$ & $-273.79(4)$ & $-274.0(9)$ \\
\hline & $-0.0062(34)$ & $-0.0111(12)$ & $-0.0083(21)$ \\
\hline & bromoethane[38] & t-1-bromopropane[40] & aa-1-bromobutane[37] \\
\hline${ }^{81}{ }^{\mathrm{Br}} \chi_{z z}(\mathrm{MHz})$ & $453.91(203)$ & $451.9(7)$ & $453.95(35)$ \\
\hline${ }^{81}{ }^{8 r} \chi_{y y}(\mathrm{MHz})$ & $-225.25(201)$ & $223.1(7)$ & $-225.5(7)$ \\
\hline${ }^{81} \mathrm{Br} \chi_{x x}(\mathrm{MHz})$ & $-228.65(18)$ & $-228.77(4)$ & $-228.4(9)$ \\
\hline$\eta_{\chi}$ & $-0.0075(48)$ & $-0.0125(16)$ & $-0.0065(24)$ \\
\hline
\end{tabular}

${ }^{a}$ Numbers in parentheses give standard errors $(1 \sigma, 67 \%$ confidence level) in units of the least significant figure.

${ }^{b} \eta_{\chi}$ is a measure of the asymmetry of the nuclear quadrupole coupling tensor, where $\eta_{\chi}=\frac{\chi_{x x}-\chi_{y y}}{\chi_{z z}}$. 
three conformational isomers, resulting from differences in the $\mathrm{I}-\mathrm{C}-\mathrm{C}-\mathrm{C}$ and $\mathrm{C}-\mathrm{C}-\mathrm{C}-\mathrm{C}$ dihedral angles, do not translate to substantial variations in the chemical environment at the iodine nucleus. The most meaningful quantification of the differences between these chemical environments or more specifically, the electric field gradient at the iodine nucleus, can be made through a comparison of the various diagonalized nuclear quadrupole coupling tensors, $\chi$, where $\chi$ is a projection of the NQC tensor in the principal axis system of the quadrupolar nucleus (e.g. iodine), as opposed to a projection of the tensor in the inertial axis system of the molecule. The advantage of comparing these diagonalized tensors is that they are all projections into an axis system which is independent of the conformation. Table 5 presents the diagonalized $\chi$ tensor of iodine in each conformer. Diagonalization was performed with QDIAG[29]. It should be noted that the $\chi_{z z}$ element is the best means of comparison because it is the projection of $\chi$ onto the z-axis of the nuclear quadrupole, which is pointed nearly along the $\mathrm{C}-\mathrm{I}$ bond, whereas the orientation of the $\mathrm{x}$ - and $\mathrm{y}$-axis are not known. While the differences in the rotated NQC tensor elements of the three conformers are small, they are never the less significant, as can be seen in Table 5. Upon inspection, only a $3.15 \%$ difference between $\chi_{z z}$ of the gg- and aaspecies is observed, even though the geometry of the alkane chain is drastically different in each case. Even smaller differences in $\chi_{z z}$, of $2.56 \%$ and $0.64 \%$, are present when comparing the remaining two pairs of conformers, namely the ggversus the ga-species, and the ga- versus the aa-species, respectively. This is purely a reflection of the fact that the two latter pairs of conformers possess more similar geometries than the first pair that was mentioned. This indicates that there is hardly a change in the electric field gradient at the iodine nucleus upon rather significant conformational changes.

Similar comparisons, based on a study by Kim et al., between the $\chi_{z z}$ element ${ }_{185}$ of $\chi$ belonging to both ${ }^{79} \mathrm{Br}$ and ${ }^{81} \mathrm{Br}$ in the gg-, ga-, and aa-species of 1 bromobutane were made[37]. These tensors are presented in Table 6. At most, a $0.7 \%$ difference between the ${ }^{81} \mathrm{Br}$ gg- and aa-species was determined, which is just over four and a half times less than the percent difference found between 
the iodine gg- and aa-species. The greater differences between the $\chi_{z z}$ elements

of the 1-iodobutane conformers is as expected and can be explained simply by the fact that iodine is both larger and more polarizable than bromine[41].

Additional comparisons were made between progressively longer haloalkanes, namely iodoethane, t-1-iodopropane, aa-1-iodobutane, and the corresponding bromine analogs. These NQCCs are presented in Table 7. In order to complete

195 Table 7, the NQCCs belonging to t-1-bromopropane were measured by highresolution microwave spectroscopy because these values could not be found in the previous work on this species by Sarachman[42]. Table 7 shows clearly that the NQC tensors of iodine in each of these progressively longer haloalkanes are identical, within experimental error. Unsurprisingly, additional terminal carbon atoms seem to have a very small effect on the electric field gradient at the iodine nucleus. Upon investigating the differences in the bromine analogs, the same conclusion can be realized. Although this does not necessarily present itself as a surprise, one curious trend did emerge. If closer attention is payed to Table 7 , it can be seen that although the tensors amongst alkanes with identical halogen substituents are the same, within experimental error, the tensors belonging to iodine, ${ }^{79} \mathrm{Br}$, and ${ }^{81} \mathrm{Br}$ are in better agreement between the ethane and butane chains than between the ethane and propane chains or the propane and butane chains. However, this trend remains inconclusive because the NQCCs belonging to ${ }^{35} \mathrm{Cl}$ and ${ }^{37} \mathrm{Cl}$ in aa-1-chlorobutane have yet to be obtained. Additionally, 210 NQC tensors belonging to longer iodine- and bromine-containing alkanes have also yet to be measured, due to the fact that collecting and measuring spectra for progressively longer haloalkanes becomes more and more challenging.

\section{Conclusion}

The reinvestigation of 1-iodobutane by high-resolution microwave spectroscopy in the frequency range of $7-13 \mathrm{GHz}$ led to the determination of rotational constants, centrifugal distortion constants, nuclear quadrupole coupling constants, and nuclear-spin rotation constants for three low energy conformations. The 
full NQC tensor of iodine in each conformation was obtained, which allowed for comparisons to be made between the chemical environments of these conformers, as well as other similar haloalkanes.

\section{Acknowledgments}

The authors thank Professor Wallace (Pete) Pringle for many useful discussions and Professor W. C. Bailey for the calculations that he shared. This work was supported at Wesleyan University by NSF grant CHE-1565276. The cluster at Wesleyan University is supported by the NSF under CNS-0619508. The Pacific Northwest National Laboratory is operated for the United States Department of Energy by the Battelle Memorial Institute under contract DEAC05-76RLO 1830.

\section{Supplemental Material}

The final fit outputs for gg-, ga-, and aa-1-iodobutane are provided.

\section{References}

[1] C. Townes, B. Dailey, J. Chem. Phys. 17 (1949) 782-796.

[2] W. Gordy, R. L. Cook, Microwave Molecular Spectra, 1984, Wiley, New York, 1984.

235

[3] S. E. Novick, J. Mol. Spectrosc. 267 (2011) 13-18.

[4] D. A. Obenchain, D. S. Frank, S. E. Novick, W. Klemperer, J. Chem. Phys. 143 (2015) 084301.

[5] H. O. Leung, J. Chem. Phys. 107 (1997) 2232-2241.

[6] H. O. Leung, A. M. Osowski, O. A. Oyeyemi, J. Chem. Phys. 114 (2001) 4829-4836.

[7] H. O. Leung, Chem. Commun. (1996) 2525-2526. 
[8] H. O. Leung, D. Gangwani, J.-U. Grabow, J. Mol. Spectrosc. 184 (1997) $106-112$.

[9] H. O. Leung, J. Chem. Phys. 110 (1999) 4394-4401. doi:10.1063/1. 3517494.

[10] H. O. Leung, O. M. Ibidapo, P. I. Abruña, M. B. Bianchi, J. Mol. Spectrosc. $222(2003) 3-14$.

[11] H. O. Leung, J. Chem. Phys. 108 (1998) 3955-3961.

[12] M. D. Marshall, H. O. Leung, J. Mol. Spectrosc. 196 (1999) 149-153.

[13] H. O. Leung, W. T. Cashion, K. K. Duncan, C. L. Hagan, S. Joo, J. Chem. Phys. 121 (2004) 237-247.

[14] M. S. NgarıİČ, W. Jäger, J. Mol. Spectrosc. 192 (1998) 320-330.

[15] M. S. Ngarıİ̌, W. Jäger, J. Chem. Phys. 111 (1999) 3919-3928.

[16] N. R. Walker, A. J. Minei, S. E. Novick, A. C. Legon, J. Mol. Spectrosc. 251 (2008) 153-158.

[17] P. Pyykkö, Mol. Phys. 106 (2008) 1965-1974.

[18] IUPAC, Quantities, units and symbols in physical chemistry, 2007.

[19] J. L. Neill, S. T. Shipman, L. Alvarez-Valtierra, A. Lesarri, Z. Kisiel, B. H. Pate, J. Mol. Spectrosc. 269 (2011) 21-29.

[20] E. A. Arsenault, D. A. Obenchain, Y. J. Choi, T. A. Blake, S. A. Cooke, S. E. Novick, J. Phys. Chem. A 120 (2016) 7145-7151.

[21] G. G. Brown, B. C. Dian, K. O. Douglass, S. M. Geyer, S. T. Shipman, B. H. Pate, Rev. Sci. Instrum. 79 (2008) 053103.

[22] G. S. Grubbs II, C. T. Dewberry, K. C. Etchison, K. E. Kerr, S. A. Cooke, Rev. Sci. Instrum. 78 (2007) 096106. 
[23] D. Feller, J. Comput. Chem. 17 (1996) 1571-1586.

[24] K. L. Schuchardt, B. T. Didier, T. Elsethagen, L. Sun, V. Gurumoorthi, J. Chase, J. Li, T. L. Windus, J. Chem. Inf. Model. 47 (2007) 1045-1052.

[25] M. J. Frisch, G. W. Trucks, H. B. Schlegel, G. E. Scuseria, M. A. Robb, J. R. Cheeseman, G. Scalmani, V. Barone, B. Mennucci, G. A. Petersson, H. Nakatsuji, M. Caricato, X. Li, H. P. Hratchian, A. F. Izmaylov, J. Bloino, G. Zheng, J. L. Sonnenberg, M. Hada, M. Ehara, K. Toyota, R. Fukuda, J. Hasegawa, M. Ishida, T. Nakajima, Y. Honda, O. Kitao, H. Nakai, T. Vreven, J. A. Montgomery, Jr., J. E. Peralta, F. Ogliaro, M. Bearpark, J. J. Heyd, E. Brothers, K. N. Kudin, V. N. Staroverov, R. Kobayashi, J. Normand, K. Raghavachari, A. Rendell, J. C. Burant, S. S. Iyengar, J. Tomasi, M. Cossi, N. Rega, J. M. Millam, M. Klene, J. E. Knox, J. B. Cross, V. Bakken, C. Adamo, J. Jaramillo, R. Gomperts, R. E. Stratmann, O. Yazyev, A. J. Austin, R. Cammi, C. Pomelli, J. W. Ochterski, R. L. Martin, K. Morokuma, V. G. Zakrzewski, G. A. Voth, P. Salvador, J. J. Dannenberg, S. Dapprich, A. D. Daniels, Ã. Farkas, J. B. Foresman, J. V. Ortiz, J. Cioslowski, D. J. Fox, Gaussian-09 revision d.01, 2013. Gaussian Inc. Wallingford, CT 2009.

[26] Z. Kisiel, L. Pszczółkowski, I. R. Medvedev, M. Winnewisser, F. C. De Lucia, E. Herbst, J. Mol. Spectrosc. 233 (2005) 231-243. URL: http: //www.sciencedirect.com/science/article/pii/S0022285205001554. doi:10.1016/j.jms. 2005.07.006.

[27] H. M. Pickett, J. Mol. Spectrosc. 148 (1991) 371-377. URL: http://www. sciencedirect.com/science/article/pii/0022285291903930. doi:10. 1016/0022-2852 (91) 90393-0.

[28] S. E. Novick, J. Mol. Spectrosc. 329 (2016) 1-7.

[29] Z. Kisiel, PROSPE-Programs for ROtational SPEctroscopy, 2000. 
[30] W. C. Bailey, Calculation of nuclear quadrupole coupling constants in gaseous state molecules, http://nqcc.wcbailey.net/, 2010.

[31] W. C. Bailey, Nuclear quadrupole coupling constants in 1-iodobutane, http://nqcc.wcbailey.net/, 2016.

[32] W. C. Bailey, Private communication, 2016.

[33] P. Thaddeus, L. Krisher, J. Loubser, J. Chem. Phys. 40 (1964) 257-273.

[34] D. Posener, Austr. J. Phys. 11 (1958) 1-17.

[35] D. Boucher, J. Burie, D. Dangoisse, J. Demaison, A. Dubrulle, J. Chem. Phys. 29 (1978) 323-330.

[36] W. E. Steinmetz, F. Hickernell, I. K. Mun, L. H. Scharpen, J. Mol. Spectrosc. 68 (1977) 173-182.

[37] J. Kim, H. Jang, S. Ka, D. A. Obenchain, R. A. Peebles, S. A. Peebles, J. J. Oh, J. Mol. Spectrosc. 328 (2016) 50-58.

[38] T. Inagusa, M. Fujitake, M. Hayashi, J. Mol. Spectrosc. 128 (1988) 456-468.

[39] M. Fujitake, M. Hayashi, J. Mol. Spectrosc. 127 (1988) 112-124.

[40] E. A. Arsenault, S. E. Novick, Private communication, 2016.

[41] E. V. Anslyn, D. A. Dougherty, Modern Physical Organic Chemistry, University Science Books, 2006.

[42] T. Sarachman, in: International Symposium on Molecular Spectroscopy, Ohio State University, 1968, p. N7. 


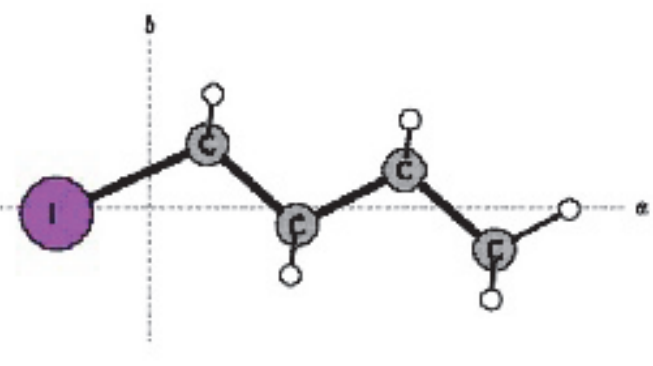

anti-anti

(aa)

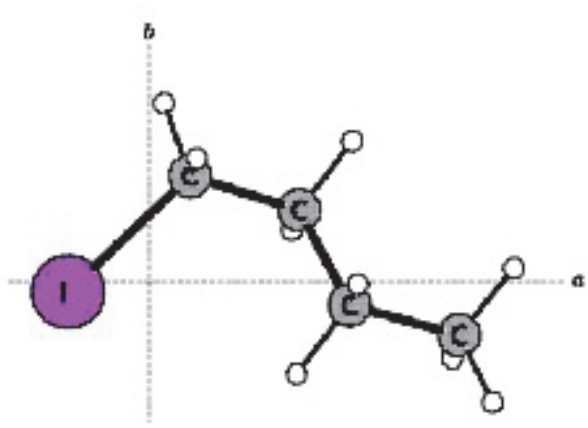

gauche-anti

(ga)

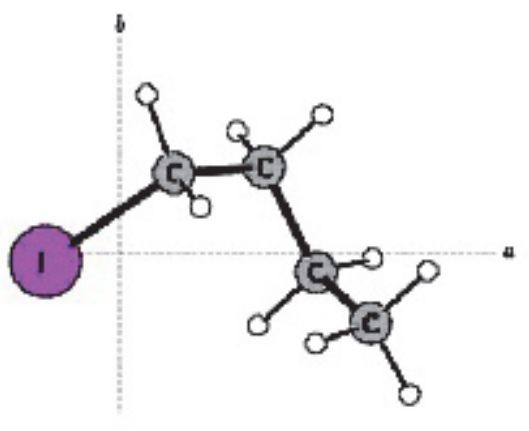

gauche-gauche (gg) 